\title{
Black holes and large $N$ complex saddles in 3D-3D correspondence
}

\author{
Sunjin Choi, ${ }^{a, b}$ Dongmin Gang ${ }^{a, c, d}$ and Nakwoo Kim ${ }^{b, e}$ \\ ${ }^{a}$ Department of Physics and Astronomy \& Center for Theoretical Physics, \\ Seoul National University, \\ 1 Gwanak-ro, Seoul 08826, Republic of Korea \\ ${ }^{b}$ School of Physics, Korea Institute of Advanced Study, \\ 85 Hoegi-ro, Dongdaemun-gu, Seoul 02455, Republic of Korea \\ ${ }^{c}$ Asia Pacific Center for Theoretical Physics (APCTP), \\ 77 Cheongam-ro, Pohang 37673, Republic of Korea \\ ${ }^{d}$ Department of Physics, Pohang University of Science and Technology (POSTECH), \\ 77 Cheongam-ro, Pohang 37673, Republic of Korea \\ ${ }^{e}$ Department of Physics and Research Institute of Basic Science, Kyung Hee University, \\ 26 Kyungheedae-ro, Dongdaemun-gu, Seoul 02447, Republic of Korea \\ E-mail: csj37100@snu.ac.kr, arima275@snu.ac.kr, nkim@khu.ac.kr
}

Abstract: We study the large $N$ sign oscillation of the twisted indices for 3D theories of class $\mathcal{R}$ obtained from M5-branes wrapped on a hyperbolic 3-manifold. Holographically, the oscillatory behavior can be understood from the imaginary part of on-shell actions for the two Euclidean supergravity solutions, Bolt \pm with $p=0$, which are Wick rotation of magnetically charged $\mathrm{AdS}_{4}$ black holes. The two solutions have the same imaginary part with opposite sign. The imaginary part comes from the $F \wedge F$-term in the supergravity and the coefficient is proportional to the Chern-Simons invariant of 3-manifold. Combining the holographic computation with 3D-3D relation for twisted indices, we propose a non-trivial mathematical conjecture regarding the phase factor of a twisted Reidemeister-Ray-Singer torsion on hyperbolic 3-manifold.

Keywords: 1/N Expansion, Black Holes in String Theory, M-Theory, Supersymmetry and Duality

ARXIV EPRINT: 2012.10944 


\section{Contents}

1 Introduction and summary 1

2 Large $N$ complex saddles in AdS/CFT 3

2.1 Conformal Field Theory side 3

2.2 AdS gravity side 4

3 Complex saddles in 3D-3D correspondence 5

$3.13 \mathrm{D}$ theory $T_{N}[M]$ from wrapped M5-branes 6

3.2 3D-3D relations for twisted indices 6

3.3 Two dominant $\mathrm{SL}(N, \mathbb{C})$ flat connections at large $N \quad 7$

3.4 Supergravity analysis 9

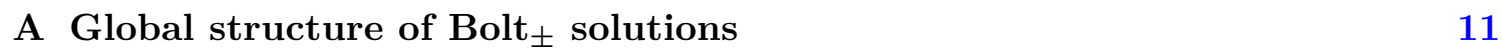

B Numerical verification of the conjecture (3.21) with $M=\left(S^{3} \backslash 4_{1}\right)_{P / Q} \quad 12$

\section{Introduction and summary}

AdS/CFT correspondence provides a consistent non-perturbative definition of quantum gravity in asymptotically AdS spacetimes [1]. The correspondence is expected to provide a systematic way to understand microscopic physics of black holes, which are key research objects in quantum gravity. One immediate and important application is to statistically account for the Bekenstein-Hawking formula of the black hole entropy from the field theory. After an early attempt using superconformal index in [2], it has long been believed that the index cannot account for the exponentially growing behavior of the microstates of black hole due to huge cancellation between bosonic/fermionic states. The situation began to change after the works of $[3,4]$, where they obtained an exponentially growing behavior from another type of BPS index called topologically twisted indices [4-6]. The exponential growth at leading order in $1 / N$ expansion nicely matches with the expected behavior from Bekenstein-Hawing entropy formula of dyonic black holes. The holographic computation was done using the $\mathrm{AdS}_{4} / \mathrm{CFT}_{3}$ associated to the M2-branes and after that the work was extended to various types of $\mathrm{AdS}_{4} / \mathrm{CFT}_{3}$ examples. Finally, the entropies of electrically charged rotating AdS black holes were also reproduced [7-10] from a large $N$ limit on superconformal index of dual field theory by revisiting the computation in [2]. More recently, it was shown that one can study not only the absolute degeneracy saturating the BekensteinHawking entropy from the superconformal index but also the sign oscillation of the index through the complex conjugate pair of large $N$ saddle points of the Legendre transformation of the free energy [11]. In the dual gravity side, these conjugate large $N$ saddles 
correspond to the complex conjugate pair of the Euclidean solutions, which are the Wick rotated version of the electrically charged rotating $\mathrm{AdS}_{5}$ BPS black hole solutions $[12,13]$.

In this paper, we study the sign oscillation of $(2+1) \mathrm{D}$ topologically twisted indices, which holographically count, with sign, microstates of magnetically charged $\mathrm{AdS}_{4}$ black holes. For the purpose, we study a particular class of $\mathrm{AdS}_{4} / \mathrm{CFT}_{3}$ correspondence arising from $N$ M5-branes wrapped on a compact hyperbolic 3-manifold $M$. The superconformal field theories are often called 3D theories of class $\mathcal{R}$. One nice feature of these 3D superconformal field theories is that we can use the 3D-3D correspondence to compute various supersymmetric quantities from $\operatorname{SL}(N, \mathbb{C})$ Chern-Simons theory invariants of $M$ [14-16]. In [17], using the 3D-3D correspondence, the large $N$ limit of the topologically twisted index $I_{g}$ on Riemann surface $\Sigma_{g}$ of genus $g>1$ was computed and its large $N$ exponential growth was shown to agree with the Bekenstein-Hawking entropy of magnetic BPS black holes in the $\mathrm{AdS}_{4}$ dual with $\mathrm{AdS}_{2} \times \Sigma_{g}$ near-horizon geometry [18, 19]. Standard lore is that bosonic states always dominate in the microstates of the magnetic BPS black holes and thus the twisted indices are always positive at sufficiently large $N$ [20]. In contrast, we will see that there exists sign oscillation in the twisted indices of class $\mathcal{R}$ theories at large $N$. The sign oscillation can be studied in two independent ways. First, using the 3D-3D correspondence, we demonstrate that the large $N$ limit of the twisted index receives contributions from a pair of the equally dominant complex conjugate saddles, exhibiting the sign oscillation as $N$ or $g$ varies. Such sign oscillation is governed by the Chern-Simons invariant of the hyperbolic 3-manifold $M, \operatorname{cs}(M)$, as following:

$$
\begin{aligned}
I_{g}\left[T_{N}[M]\right] & \sim \exp \left((g-1) \frac{\operatorname{vol}(M)+i c s(M)}{3 \pi} N^{3}+\mathcal{O}(N)\right)+(c . c .) \\
& =\exp \left((g-1) \frac{\operatorname{vol}(M)}{3 \pi} N^{3}+\mathcal{O}(N)\right) \cos \left((g-1) \frac{c s(M)}{3 \pi} N^{3}+\mathcal{O}(N)\right) .
\end{aligned}
$$

Here, $\operatorname{vol}(M)$ is the hyperbolic volume. The exponentially growing factor equals the Bekenstein-Hawking entropy of the magnetically charged BPS black holes in the $\mathrm{AdS}_{4}$ dual, i.e. $S_{\mathrm{BH}}=(g-1) \frac{\operatorname{vol}(M)}{3 \pi} N^{3}$ [21]. Next, we compute the (regularized) Euclidean onshell action in the $\mathrm{AdS}_{4}$ gravity dual. After the Wick rotation of the magnetic BPS black hole, the Euclidean solutions come in a pair; the Bolt ${ }_{ \pm}$solutions with $p=0$ and $g>1$ [22]. While Bolt \pm solutions are purely real, we show that their contributions to the Euclidean on-shell action are complex conjugate to each other. The imaginary part of the on-shell action comes from the topological $\theta$-term in $4 \mathrm{D} \mathcal{N}=2$ minimal gauged supergravity whose bosonic action in the Euclidean signature reads

$$
S=-\frac{1}{16 \pi G_{4}} \int d^{4} x \sqrt{g}\left(R+\frac{6}{L^{2}}-F_{\mu \nu} F^{\mu \nu}\right)+i \frac{\theta}{4 \pi^{2} L^{2}} \int F \wedge F,
$$

where $G_{4}$ is the $4 \mathrm{D}$ Newton's constant and $L$ is the $\mathrm{AdS}_{4}$ radius. Such purely imaginary $\theta$-term is often overlooked in the literature. The above $4 \mathrm{D}$ action can be derived from the consistent KK truncation of 7D maximal gauged supergravity on $M$ [23-25]. Also, the 7D gauged supergravity can be uplifted to $11 \mathrm{D}$ supergravity on a squashed 4-sphere $\tilde{S}^{4}[26-$ 28]. As the $3 \mathrm{D}$ class $\mathcal{R}$ theory is holographically dual to the M-theory on $\operatorname{AdS}_{4} \times M \times \tilde{S}^{4}$, 
such successive embedding relates the macroscopic parameters $L, G_{4}, \theta$ to the microscopic parameters $N, \operatorname{vol}(M), c s(M)$ as following:

$$
\frac{L^{2}}{G_{4}}=\frac{N^{3}+\mathcal{O}(N)}{6} \frac{4 \operatorname{vol}(M)}{\pi^{2}}, \quad \theta=-\frac{N^{3}+\mathcal{O}(N)}{6} \frac{2 \operatorname{cs}(M)}{\pi} .
$$

Here, $\mathcal{O}(N)$ refers to a quantum shift, which is difficult to determine from the supergravity analysis. With these relations and appropriate counterterms needed for the holographic renormalization $[20,22]$, the Euclidean on-shell action evaluated on Bolt \pm with $p=0$ are given by

$$
S\left[\text { Bolt }_{ \pm}\right]=(1-g) \frac{N^{3}+\mathcal{O}(N)}{6} \frac{2}{\pi}(\operatorname{vol}(M) \pm i c s(M)),
$$

which is consistent with the field theory computation (1.1) at the leading $N^{3}$ order. Here, $\operatorname{vol}(M) \pm i \operatorname{cs}(M)$ is the complex volume, which is the holomorphic topological invariant of the hyperbolic 3-manifold $M$ [29]. The invariant is well-defined only modulo $i \pi^{2}$. For the exponentiated classical action $e^{-S\left[\text { Bolt }_{ \pm}\right]}$to be well-defined, the undetermined quantum shift $\mathcal{O}(N)$ should satisfy following condition:

$$
\frac{N^{3}+\mathcal{O}(N)}{6} \in \mathbb{Z}
$$

Note that the $\theta$-term in (1.2) does not appear from the consistent truncation of 11D supergravity on the Sasaki-Einstein 7 -manifold $\left(\mathrm{SE}_{7}\right)$. That is the reason why we did not have to consider such term when we analyze the black holes in $\mathrm{AdS}_{4} \times \mathrm{SE}_{7}$ dual to M2brane SCFTs [22]. Also, the on-shell value of the $\theta$-term vanishes if there is no topological twist on $\mathrm{AdS}_{4}$, so it does not affect the on-shell action of the electrically charged rotating BPS black holes in $\mathrm{AdS}_{4}$ regardless of embedding in 11D supergravity [13].

The rest of this paper is organized as follows. In section 2, we review complex saddles and sign oscillation of $4 \mathrm{D}$ superconformal index in the large $N$ limit. In section 3 , we study the sign oscillation in the large $N$ twisted indices of 3D class $\mathcal{R}$ theories from both of 3D-3D dual complex Chern-Simons theory and holographic dual supergrvity. As a byproduct of the analysis, we propose a concrete mathematical conjecture in (3.21), which is numerically confirmed in appendix B.

\section{Large $N$ complex saddles in AdS/CFT}

In this section, we briefly review basic ideas about the large $N$ complex saddle points $[7,10-$ $13,30]$ associated to the electrically charged rotating AdS black holes. In the dual conformal field theory side, the microstates of black holes correspond to the local operators with fixed macroscopic charges.

\subsection{Conformal Field Theory side}

Following [11], our concrete example is the superconformal index [2,31] of $4 \mathrm{D} \mathcal{N}=4$ supersymmetric-Yang-Mills theory with $\mathrm{U}(N)$ gauge group, which is dual to the type IIB 
string theory on $\mathrm{AdS}_{5} \times S^{5}$. The (unrefined) superconformal index is defined as

$$
Z=\operatorname{Tr}_{\mathcal{H}_{\mathrm{rad}}\left(S^{3}\right)}\left[(-1)^{F} x^{3(R+2 J)}\right]=\sum_{j=0}^{\infty} \Omega_{j} x^{j},
$$

where $R$ is the superconformal R-charge, $J=\frac{J_{1}+J_{2}}{2}$ with $J_{i}$ 's being two angular momenta on $S^{3}$, and $j \equiv 3(R+2 J)$. The trace is taken over the radially quantized Hilbert space $\mathcal{H}_{\text {rad }}\left(S^{3}\right)$ on $S^{3} \times \mathbb{R}$ whose states correspond to local operators on $\mathbb{R}^{4}$. The above index receives contributions only from the BPS states with $j \geq 0$ and $\Omega_{j}$ counts the $(-1)^{F_{-}}$ weighted number of BPS states with the charge $j$. In the large $N$ limit, one may compute the free energy $\log Z \sim \mathcal{O}\left(N^{2}\right)$ using the saddle point approximation, where the large $N$ saddle points are supposed to correspond to the black hole saddle points in the $\mathrm{AdS}_{5}$ gravity dual. In order to extract the entropy from the large $N$ free energy, one should perform the Legendre transformation to the microcanonical ensemble, which is the saddle point approximation of the inverse Laplace transformation

$$
\Omega_{j}=\frac{1}{2 \pi i} \oint \frac{d x}{x} x^{-j} Z(x)
$$

at macroscopic charge $j \sim \mathcal{O}\left(N^{2}\right)$. A known fact is that the dominant saddle point values $x_{*}$ of $x$ are complex at real positive charge $j$. Naively, this yields the complex entropy $S(j)$ at real charge. However, as pointed out in $[9,11]$, the unitarity of our QFT guarantees that there always exists a complex conjugate saddle point for any such complex saddle point. Then, adding two equivalently dominant contributions from the complex conjugate pair of saddle points, we obtain

$$
\Omega_{j} \sim e^{S(j)}+e^{\overline{S(j)}} \sim \exp [\operatorname{Re}(S(j))+\cdots] \cos [\operatorname{Im}(S(j))+\cdots],
$$

where $\cdots$ denote possible subleading corrections in the large $N$ expansion. In $[11,32]$, they numerically studied the index of $\mathcal{N}=4 \mathrm{SYM}$, and in [11] it was shown that the above formula is matched very nicely by the numerically computed index at reasonably large $N$ and $j$. The first macroscopic exponential factor captures the Bekenstein-Hawking entropy of electrically charged rotating BPS black holes in $\mathrm{AdS}_{5} \times S^{5}[33-36]$, i.e. $\operatorname{Re}(S(j))=S_{\mathrm{BH}}$. The second oscillating cosine factor accounts for the sign oscillation of $\Omega_{j}$ as $j$ varies due to the $(-1)^{F}$ factor [11].

In the above example, the complex conjugate pair of saddle points of the Legendre transformation is to imitate the sign oscillation of $\Omega_{j}$ of the index by the cosine function. This structure is universal and should be realized in any supersymmetric index on $\mathcal{M}_{D-1} \times$ $S^{1}$ of the $D$-dimensional unitary QFT, if the index exhibits overall sign oscillation. In particular, one can easily check that the superconformal indices of SCFTs in $D=3[9,37$ $39], D=4[7,8,40,41], D=5[10,42]$, and $D=6[7,43]$ exhibit such complex saddle points of the Legendre transformation at large $N$. (There have been many works on this subject in the recent years. Please refer to the references of the above for further details.)

\section{$2.2 \quad$ AdS gravity side}

One may want to interpret the above large $N$ complex saddle points and the phase of the entropy in the dual gravity side. Obviously, in the Lorentzian black hole solution, there are 
no such complex quantities. The Bekenstein-Hawking entropy is just given by the horizon area of the black hole, and the chemical potentials are the electrostatic potentials and the angular velocities at the horizon. So they are all genuinely physical observables. In order to obtain complex quantities, one should Wick rotate the BPS black hole solution, and study the (regularized) Euclidean on-shell action. In [12, 13], it was shown that the electrically charged rotating BPS black hole solutions in $\mathrm{AdS}_{D+1=4,5,6,7}$ become complex as we Wick rotate them to the Euclidean solutions. Furthermore, there are always a complex conjugate pair of Euclidean solutions corresponding to one rotating Lorentzian BPS black hole solution. This conjugate pair of the Euclidean solutions naturally corresponds to the conjugate pair of the Legendre transform saddle points of the CFT index in the large $N$ limit. The extremized chemical potentials, free energy, and the entropy, which are complex, are precisely matched between each solution of the conjugate pair [13].

\section{Complex saddles in $3 \mathrm{D}-3 \mathrm{D}$ correspondence}

In this section, we consider the universal magnetically charged $\mathrm{AdS}_{4}$ BPS black holes in $4 \mathrm{D}$ minimal $\mathcal{N}=2$ gauged supergravity $[18,19]$. Its bosonic action in the Euclidean signature is given in (1.2). There are two well-known ways of embedding this low energy effective supergravity into a full fledged quantum gravity theory, M-theory. One is using multiple M2-branes probing a cone over Sasaki-Einstein 7-manifold $Y_{7}$, and the other is using multiple M5-branes wrapped on a compact 3-manifold $M$. The macroscopic constants, $G_{4}$ and $\theta$, in the $4 \mathrm{D}$ supergravity are determined by the microscopic data, $N$ (the number of branes) and geometric quantities of the internal manifold ( $Y_{7}$ or $\left.M\right)$. The $\theta$-term turns out to vanish, i.e. $\theta=0$, for the $4 \mathrm{D}$ supergravity from multiple M2-branes, while it does not vanish for the case of multiple M5-branes. The $\theta$-term has been usually overlooked in the literature, but here we point out that the $\theta$-term naturally appears in the consistent KK truncation of the chiral 7D maximal gauged supergravity [23-25], which is holographically dual to the $6 \mathrm{D}(2,0)$-theory living on the M5-branes, to the 4D minimal gauged supergravity. Moreover, we demonstrate that the $\theta$-angle is proportional to a topological invariant called the 'Chern-Simons invariant', $c s(M)$, of the internal 3-manifold.

The non-trivial $\theta$-term has interesting implications in the large $N$ analysis of the twisted indices of the dual $3 \mathrm{D} \mathcal{N}=2$ superconformal field theory. It determines the sign oscillatory behavior of the twisted indices at large $N$. After the Wick rotation, the magnetically charged black holes in $\mathrm{AdS}_{4}$ become a pair of Euclidean saddle points called Bolt $_{ \pm}$with $p=0$ and $g>1[22]$. The $\theta$-term contributes as an imaginary part to the Euclidean action and the on-shell actions for Bolt \pm are complex and conjugate to each other. At large $N$, the Bolt \pm solutions give the most dominant contributions to the path integral of the $\mathrm{AdS}_{4}$ quantum gravity with an asymptotic boundary condition associated to the twisted indices. Then, the imaginary part of their on-shell action determine the oscillatory phases factor of the twisted indices at large $N$. See (2.3) for the similar oscillatory phase factor for electrically charged black holes and superconformal indices [11].

The supergravity computation of $\theta$-term also has interesting mathematical implications. Combining the holographic principle and 3D-3D relation for twisted indices, the Eu- 
clidean saddles Bolt \pm are mapped to two canonical $\operatorname{SL}(N, \mathbb{C})$ flat connections, $\mathcal{A}_{N}^{\text {geom }}$ and $\mathcal{A}_{N}^{\overline{\text { geom }}}$, on the internal hyperbolic 3 -manifold $M$. The on-shell values of $\theta$-term for Bolt \pm determine the large $N$ behavior of the phase factor of a topological invariant called the adjoint Reidemeister-Ray-Singer torsion associated to the two $\operatorname{SL}(N, \mathbb{C})$ flat connections.

\subsection{D theory $T_{N}[M]$ from wrapped M5-branes}

Here, we briefly review the $3 \mathrm{D} T_{N}[M]$ theory geometrically constructed from wrapped $N$ M5-branes on the compact 3-manifold $M[14,16,44,45]$. The theory can be obtained from a twisted compactification of $6 \mathrm{D} A_{N-1}(2,0)$-theory, which is the world-volume theory of $N$ M5-branes:

$$
6 \mathrm{D} A_{N-1}(2,0) \text {-theory on } \mathbb{R}^{1,2} \times M \longrightarrow 3 \mathrm{D} T_{N}[M] \text { theory on } \mathbb{R}^{1,2} \text {. }
$$

In the compactification, we perform a partial topological twisting along the compact 3manifold $M$ to preserve some supersymmetries. In the topological twisting, we use the $\mathrm{SO}(3)$ subgroup of $6 \mathrm{D} \mathrm{SO}(5)$ R-symmetry and the twisting preserves 4 supercharges out of 16 . Thus, the resulting $T_{N}[M]$ theory has $3 \mathrm{D} \mathcal{N}=2$ supersymmetry. Field theoretic construction of the $T_{N}[M]$ theory is given in $[16,44,45]$. From the explict construction, one can confirm that the $T_{N}[M]$ only has U(1) R-symmetry and no other flavor symmetry at sufficiently large $N$ for hyperbolic $M$. The U(1) R-symmetry originates from the $\mathrm{SO}(2)$ subgroup of $6 \mathrm{D} \mathrm{SO(5)} \mathrm{R-symmetry} \mathrm{and} \mathrm{thus} \mathrm{the} \mathrm{R-charge} \mathrm{is} \mathrm{integer-quantized:}$

$$
R(\mathcal{O}) \in \mathbb{Z}, \text { for all local operators } \mathcal{O} \text { in } T_{N}[M] \text { theory . }
$$

Since there is no flavor symmetry to mix, the U(1) R-symmetry is identical to the IR superconformal R-symmetry.

\section{$3.2 \quad 3 \mathrm{D}-3 \mathrm{D}$ relations for twisted indices}

One nice feature of the 3D $T_{N}[M]$ theory is that the supersymmetric partition functions of the theory can be written in terms of invariants of $\operatorname{SL}(N, \mathbb{C})$ Chern-Simons theory on $M$. The simplest 3D-3D relation is the one for twisted indices. The twisted indices of $T_{N}[M]$ theory on genus $g$ Riemann surface $\Sigma_{g}$ is defined as

$$
\begin{aligned}
I_{g}\left[T_{N}[M]\right] & :=\operatorname{Tr}_{\mathcal{H}\left(\Sigma_{g}\right)}(-1)^{R}, \quad \text { where } \\
\mathcal{H}\left(\Sigma_{g}\right) & :=\text { Topologically twisted Hilbert-space on } \Sigma_{g} .
\end{aligned}
$$

Along $\Sigma_{g}$, we perform a topological twisting using the compact U(1) R-symmetry. The twisting is possible only when the following Dirac quantization is satisfied:

$$
R(\mathcal{O}) \times(g-1) \in \mathbb{Z}, \quad \text { for all local operators } \mathcal{O} .
$$

The condition is always satisfied for $T_{N}[M]$ theory thanks to the equation (3.2). The 3D-3D relation for the twisted indices is $[17,21]$

$$
I_{g}\left[T_{N}[M]\right]=\sum_{\rho \in \chi_{\text {irred }}(M ; N)}\left(N \operatorname{Tor}_{\mathrm{Adj}}\left(\rho_{\alpha} ; \mathrm{SL}(N, \mathbb{C})\right)\right)^{g-1}
$$


The above 3D-3D relation holds only for 3-manifold with trivial $H_{1}\left(M, \mathbb{Z}_{N}\right)$. For 3-manifold with non-trivial $H_{1}\left(M, \mathbb{Z}_{N}\right)$, the $3 \mathrm{D}-3 \mathrm{D}$ relation should be modified [39, 46]. But the modification only affects the $\log N$ corrections at large $N$. Here $\chi_{\text {irred }}(M ; N)$ is the set of irreducible $\mathrm{SL}(N, \mathbb{C})$ flat connections on $M$

$$
\chi_{\text {irred }}(M ; N)=\frac{\left\{\rho \in \operatorname{Hom}\left(\pi_{1} M \rightarrow \operatorname{SL}(N, \mathbb{C})\right): \rho \text { is irreducible }\right\}}{(\text { conjugation })} .
$$

The flat connections are classical solutions of $\operatorname{SL}(N, \mathbb{C})$ Chern-Simons theory on $M$. Conventionally, a $G$ flat connection is defined as gauge field configuration $\mathcal{A}$ of group $G$ with vanishing field strength, i.e. $d \mathcal{A}+\mathcal{A} \wedge \mathcal{A}=0$. A $G$ flat connection $\mathcal{A}$ induces a homomorphism $\rho: \pi_{1} M \rightarrow G$ via a holonomy matrix $\rho(a):=P \exp \left(\oint_{a} \mathcal{A}\right)$. The holonomy matrices fully characterize the flat connection and thus we can identify a flat connection $\mathcal{A}$ with its induced homomorphism $\rho$. Using the identification, the symbols $\mathcal{A}$ and $\rho$ are interchangeably used throughout this paper. A homomorphism $\rho \in \operatorname{Hom}\left(\pi_{1} M \rightarrow \operatorname{SL}(N, \mathbb{C})\right)$ is called irreducible if its stabilizer subgroup $\operatorname{Stab}(\rho) \subset \operatorname{SL}(N, \mathbb{C})$ defined in the following way is zero-dimensional.

$$
\operatorname{Stab}(\rho):=\left\{h \in \operatorname{SL}(N, \mathbb{C}):[h, \rho(a)]=0 \quad \forall a \in \pi_{1} M\right\} .
$$

$\operatorname{Tor}_{\text {Adj }}\left(\rho_{\alpha} ; \operatorname{SL}(N, \mathbb{C})\right)$ is a mathematical invariant called the adjoint Reidemeister-RaySinger torsion [47-49] associated to the flat connection $\rho_{\alpha}$. The invariant in general representation $R \in \operatorname{Hom}\left[G_{\mathbb{C}} \rightarrow \mathrm{GL}\left(V_{R}\right)\right]$ can be defined as follows

$$
\operatorname{Tor}_{R}\left[\mathcal{A}_{\alpha} ; G_{\mathbb{C}}\right]:=\frac{\left[\operatorname{det}^{\prime} \Delta_{1}\left(R, \mathcal{A}_{\alpha}\right)\right]^{1 / 2}}{\left[\operatorname{det}^{\prime} \Delta_{0}\left(R, \mathcal{A}_{\alpha}\right)\right]^{3 / 2}}
$$

Here $\Delta_{n}\left(R, \mathcal{A}_{\alpha}\right)$ is a Laplacian acting on $V_{R^{-}}$valued $n$-form twisted by a flat connection $\mathcal{A}_{\alpha}$ and $\operatorname{det}^{\prime}$ is the zeta function regularized determinant. In the definition, we need to choose a metric structure on $M$ but the final $\operatorname{Tor}_{R}$ turns out to be independent of the choice. When $R=$ (Adj) (adjoint representation), the invariant is related to the perturbative 1loop expansion of the complex Chern-Simons theory around the flat connection $\mathcal{A}_{\alpha}$ in the following way

$$
\begin{aligned}
& \left.\int \frac{[d(\delta \mathcal{A})]}{\text { (gauge })} \exp \left(-\frac{1}{2 \hbar} \int_{M} \operatorname{Tr}\left(\mathcal{A} d \mathcal{A}+\frac{2}{3} \mathcal{A}^{3}\right)\right)\right|_{\mathcal{A}=\mathcal{A}_{\alpha}+\delta \mathcal{A}} \\
& \stackrel{\hbar \rightarrow 0}{\longrightarrow} \frac{1}{\sqrt{\operatorname{Tor}_{\mathrm{Adj}}\left(\rho_{\alpha} ; \operatorname{SL}(N, \mathbb{C})\right)}} \exp \left(-\frac{1}{2 \hbar} \int_{M} \operatorname{Tr}\left(\mathcal{A}_{\alpha} d \mathcal{A}_{\alpha}+\frac{2}{3} \mathcal{A}_{\alpha}^{3}\right)+\mathcal{O}(\hbar)\right) .
\end{aligned}
$$

As a non-trivial consistency check of the 3D-3D relation, the integrality of the twisted indices in (3.5) were checked for various examples in $[17,39,46]$.

\subsection{Two dominant $\operatorname{SL}(N, \mathbb{C})$ flat connections at large $N$}

Here, we focus on the case where $M$ is a closed hyperbolic 3-manifold. At large $N$, the dominant contributions to the twisted index in (3.5) with $g>1$ come from the two irreducible flat connections, $\rho_{N}^{\text {geom }}$ and $\rho_{N}^{\overline{g e o m}}$, related to each other by complex conjugation. The flat connections are defined as follows

$$
\mathcal{A}_{N}^{\text {geom }}:=\tau_{N} \cdot(\omega+i e), \quad \mathcal{A}_{N}^{\overline{\text { geom }}}:=\tau_{N} \cdot(\omega-i e) .
$$


Here $\omega$ and $e$ are spin-connection and dreibein for the unique hyperbolic metric on $M$ normalized as $R_{\mu \nu}=-2 g_{\mu \nu}$. They can be regarded as so(3)-valued 1-form on $M$ and their complex combinations $(\omega \pm i e)$ define a complex conjugate pair of $\mathrm{SL}(2, \mathbb{C})$ connections. ${ }^{1}$ The hyperbolicity, $R_{\mu \nu}=-2 g_{\mu \nu}$, implies that the connections are actually flat. Via the principal embedding $\tau_{N}: \mathrm{SU}(2) \rightarrow \mathrm{SU}(N)$, the $\mathrm{SL}(2, \mathbb{C})$ flat connections are mapped to a complex conjugate pair of $\operatorname{SL}(N, \mathbb{C})$ connections, $\mathcal{A}_{N}^{\text {geom }}$ and $\mathcal{A}_{N}^{\overline{\text { geom }}}$. At large $N$, the two flat connections give the most dominant contributions to the twisted indices in (3.5) with $g>1$, i.e.

$$
\begin{aligned}
I_{g>1}\left[T_{N}[M]\right] \stackrel{N \rightarrow \infty}{\longrightarrow} & \left(N \operatorname{Tor}_{\mathrm{Adj}}\left(\rho_{N}^{\text {geom }} ; \mathrm{SL}(N, \mathbb{C})\right)\right)^{g-1}+(c . c) \\
& +(\text { exponentially smaller terms }) .
\end{aligned}
$$

We now study the large $N$ limit of the adjoint torsion $\operatorname{Tor}_{\text {Adj }}\left(\rho_{N}^{\text {geom }} ; \operatorname{SL}(N, \mathbb{C})\right)$. First, from the following branching rule

$$
\text { (Adjoint representation of } \mathrm{SU}(N))=\left(\bigoplus_{n=1}^{N-1} \tau_{2 n+1} \text { of } \mathrm{SU}(2)\right) \text {, }
$$

we have

$$
\log \operatorname{Tor}_{\text {Adj }}\left(\rho_{N}^{\text {geom }} ; \operatorname{SL}(N, \mathbb{C})\right)=\sum_{n=1}^{N-1} \log \mathbf{T o r}_{\tau_{2 n+1}}\left(\rho_{N=2}^{\text {geom }} ; \operatorname{SL}(2, \mathbb{C})\right) .
$$

In the above, the $\mathrm{SU}(2)$ is embedded into $\mathrm{SU}(N)$ via the principal embedding $\tau_{N}$ and $\tau_{2 n+1}$ is the $(2 n+1)$-dimensional irreducible representation of $\operatorname{SU}(2) . \operatorname{Tor}_{\tau}(\rho ; \operatorname{SL}(2, \mathbb{C}))$ is the Reidemeister-Ray-Singer torsion associated to the $\operatorname{SL}(2, \mathbb{C})$ flat connection $\rho$ in the representation $\tau$. Using Selberg's trace formula, it is mathematically proven that

\section{Theorem [50]:}

$$
\operatorname{Re}\left(\log \operatorname{Tor}_{\tau_{2 \mathrm{n}+1}}\left(\rho_{N=2}^{\text {geom }}, \operatorname{SL}(2, \mathbb{C})\right)\right)=\frac{1}{\pi} \operatorname{vol}(M)\left(n^{2}+n+\frac{1}{6}\right)+\operatorname{Re} \sum_{\gamma} \sum_{k=n+1}^{\infty} \log \left(1-q_{\gamma}^{k}\right) .
$$

Here $\operatorname{vol}(M)$ is the hyperbolic volume, volume measured using the unique hyperbolic metric normalized as $R_{\mu \nu}=-2 g_{\mu \nu}$. In the last term, the summation is over geodesics $\gamma$ in $M$ and $q_{\gamma}:=e^{-\ell_{\mathbb{C}}(\gamma)}$ where $\ell_{\mathbb{C}}(\gamma)$ is the complex length of $\gamma$. The complex length is defined as

$$
\operatorname{Tr}\left(\rho_{N=2}^{\text {geom }}(\gamma)\right)=2 \cosh \left(\frac{\ell_{\mathbb{C}}(\gamma)}{2}\right), \quad \operatorname{Re}\left(\ell_{\mathbb{C}}\right)>0
$$

Combining (3.13) and (3.14), we have the following large $N$ behavior of the adjoint torsion [17].

$$
\begin{aligned}
& \operatorname{Tor}_{\mathrm{Adj}}\left(\rho_{N}^{\text {geom }} ; \operatorname{SL}(N, \mathbb{C})\right) \\
& =e^{i \varphi(N, M)} \exp \left(\frac{\operatorname{vol}(M)}{6 \pi}\left(2 N^{3}-N-1\right)+\operatorname{Re} \sum_{[\gamma]} \log \text { P.E. }\left[\frac{q_{\gamma}^{N+1}-q_{\gamma}^{2}}{\left(1-q_{\gamma}\right)^{2}}\right]\right) .
\end{aligned}
$$

\footnotetext{
${ }^{1}$ More precisely, they are $\operatorname{PSL}(2, \mathbb{C})$ flat connections, which can always be uplifted to $\operatorname{SL}(2, \mathbb{C})$ flat connections.
} 
Here P.E. denotes the plethystic exponential,

$$
\text { P.E. }[f(q)]:=\exp \left(\sum_{n=1}^{\infty} \frac{1}{n} f\left(q^{n}\right)\right) \text {. }
$$

Then, using (3.11), we finally have following large $N$ behavior

$$
\begin{aligned}
I_{g}\left[T_{N}[M]\right] & \sim \exp \left((g-1) \frac{\operatorname{vol}(M)}{6 \pi}\left(2 N^{3}-N\right)+i(g-1) \varphi(N, M)+o(N)\right)+(c . c .) \\
& =\exp \left((g-1) \frac{\operatorname{vol}(M)}{6 \pi}\left(2 N^{3}-N\right)+o(N)\right) \times(2 \cos ((g-1) \varphi(N, M))) .
\end{aligned}
$$

The exponential growth of $I_{g}\left[T_{N}[M]\right]$ at large $N$ nicely matches with the BekensteinHawking entropy of the corresponding magnetically charged black hole in the gravity dual including subleading corrections [17, 21, 39, 51, 52].

In this paper, we focus on the oscillatory factor, $2 \cos ((g-1) \varphi(N, M))$, of the large $N$ twisted indices. As the main result, from holographic dual computation in the next section, we propose that

$$
\varphi(N, M) \stackrel{N \rightarrow \infty}{\longrightarrow}\left(\frac{c s(M)}{3 \pi} N^{3}+\mathcal{O}(N)\right) \quad(\bmod 2 \pi) .
$$

Here, $\operatorname{cs}(M)$ is the so-called Chern-Simons invariant of the hyperbolic 3-manifold $M$, which is defined as follows

$$
\frac{i}{2} \int_{M} \operatorname{Tr}\left(\mathcal{A}_{N=2}^{\text {geom }} \wedge d \mathcal{A}_{N=2}^{\text {geom }}+\frac{2}{3} \mathcal{A}_{N=2}^{\text {geom }} \wedge \mathcal{A}_{N=2}^{\text {geom }} \wedge \mathcal{A}_{N=2}^{\text {geom }}\right)=\operatorname{vol}(M)+i c s(M) .
$$

The invariant $c s(M)$ is defined only modulo $\pi^{2}$. The above holographic prediction combined with the 3D-3D relation (3.5) implies the following non-trivial mathematical conjecture

\section{Conjecture :}

$$
\operatorname{Im}\left(\log \operatorname{Tor}_{\tau_{2 \mathrm{n}+1}}\left(\rho_{N=2}^{\text {geom }}, \mathrm{SL}(2, \mathbb{C})\right) \stackrel{n \rightarrow \infty}{\longrightarrow} \frac{1}{\pi} \operatorname{cs}(M)\left(n^{2}+n\right)+\mathcal{O}\left(n^{0}\right) \quad(\bmod 2 \pi) .\right.
$$

Combined with the formula in (3.13), the conjecture implies the proposal in (3.19). We will confirm numerically the conjecture with an explicit example in appendix B.

The phase factor $e^{i \varphi(N, M)}$ determines the sign oscillatory behavior of the twisted indice $I_{g}\left[T_{N}[M]\right]$ as $g$ or $N$ varies. See figure 1 for the explicit example with $M=\left(S^{3} \backslash \mathbf{4}_{1}\right)_{P / Q=5}$.

\subsection{Supergravity analysis}

The holographic dual of $T_{N}[M]$ for a closed hyperbolic 3-manifold $M$ is proposed in [24]. At low energy, the gravity theory is described by the $4 \mathrm{D}$ minimal $\mathcal{N}=2$ gauged supergravity whose Euclidean action is given in (1.2) with

$$
L^{2} / G_{4}=\frac{2\left(N^{3}+\mathcal{O}(N)\right) \operatorname{vol}(M)}{3 \pi^{2}} .
$$



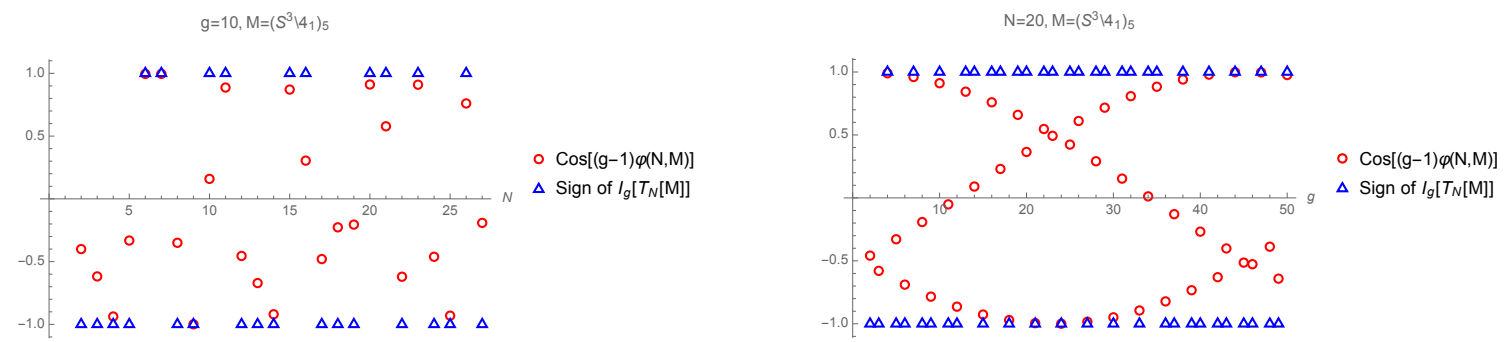

Figure 1. Sign oscillation of $I_{g}\left[T_{N}[M]\right]$ with $M=\left(S^{3} \backslash \mathbf{4}_{1}\right)_{P / Q=5}$ as $N$ varies with fixed $g=10$ (Left) or $g$ varies with fixed $N=20$ (Right). The blue triangle is the sign of $I_{g}\left[T_{N}[M]\right]$, i.e. $\frac{I_{g}\left[T_{N}[M]\right]}{\left|I_{g}\left[T_{N}[M]\right]\right|}$, while the red circle is $\cos ((g-1) \varphi(N, M))$. From the graph, one can see that twos have always the same sign. The $I_{g}\left[T_{N}[M]\right]$ is computed using the formula in (3.11) combined with (3.13) and the adjoint torsion computation summarized in appendix B. $\varphi(N, M)$ is given in (3.19) with $\operatorname{cs}(M)=1.52067$ [54]. The undetermined $\mathcal{O}(N)$ part is numerically fitted and we obtain $\varphi(N, M)=\frac{c s(M)}{3 \pi} N^{3}+0.453 N+5.01+\mathcal{O}(1 / N)$.

The 4D supergravity action can be obtained from a consistent KK truncation of 7D maximal gauged supergravity [23] on the 3-manifold $M$. In the consistently truncated 4D supergravity, there is a following topological term, which has been overlooked in the literature

$$
S_{\theta \text {-term }}=-i \frac{N^{3}+\mathcal{O}(N)}{6} \times \frac{c s(M)}{2 \pi^{3} L^{2}} \times \int F \wedge F, \quad \text { i.e. } \theta=-\frac{N^{3}+\mathcal{O}(N)}{3} \frac{c s(M)}{\pi} .
$$

Note that $\operatorname{cs}(M)$ is only defined modulo $\pi^{2}$ and $\int F \wedge F \in 4 \pi^{2} L^{2} \mathbb{Z}^{2}$ For the $\exp \left(-S_{\theta \text {-term }}\right)$ to be well-defined, we require that

$$
\frac{N^{3}+\mathcal{O}(N)}{6} \in \mathbb{Z}
$$

This gives a non-trivial consistency condition for the $\mathcal{O}\left(N^{1}\right)$ correction in $\theta$. One natural guess is

$$
\theta=-\frac{N^{3}-N}{3} \frac{\operatorname{cs}(M)}{\pi}
$$

We are going to evaluate the topological $\theta$-term on the Bolt \pm solutions with $g>1$ and $p=0$. They are Euclidean supergravity saddles obtained after Wick rotating the magnetically charged $\mathrm{AdS}_{4}$ black holes [22]. Topologically, the Euclidean solutions satisfy

$$
\begin{aligned}
\mathcal{M}_{4}^{\text {Bolt }} \pm & \sim \Sigma_{g} \times D_{2}, \\
F^{\text {Bolt }} \pm & \sim \frac{L}{2}\left(\operatorname{vol}\left(\Sigma_{g}\right) \mp \operatorname{vol}\left(D_{2}\right)\right) .
\end{aligned}
$$

Refer to appendix A for details. Here, the volume form are normalized as

$$
\begin{aligned}
\int_{\Sigma_{g}} \operatorname{vol}\left(\Sigma_{g}\right) & =4 \pi(1-g), \\
\int_{S^{2}=D_{2} \bigcup D_{2}} \operatorname{vol}\left(D_{2}\right) & =2 \times \int_{D_{2}} \operatorname{vol}\left(D_{2}\right)=4 \pi .
\end{aligned}
$$

\footnotetext{
${ }^{2}$ The bulk gauge field $A$ is normalized as $A=i L A_{\mathrm{CFT}}$, where $A_{\mathrm{CFT}}$ is the background gauge field for the compact U(1) R-symmetry in the conformal field theory at the boundary. In our convention, the boundary $A_{\mathrm{CFT}}$ is purely imaginary. The R-symmetry is normalized so that $R(Q)= \pm 1$ for supercharges $Q$.
} 
At the boundary $\partial\left(\mathcal{M}_{4}^{\text {Bolt }}{ }_{ \pm}\right)=\Sigma_{g} \times S^{1}$, the gauge field configuration is given by

$$
F_{\mathrm{CFT}}^{\mathrm{Bolt} \pm}=\frac{1}{2 i} \operatorname{vol}\left(\Sigma_{g}\right) \mp \frac{1}{2 i} \operatorname{vol}\left(D_{2}\right) .
$$

The first term is compatible with the fact that there is topological twisting on $\Sigma_{g}$ and the second term is compatible with the factor $(-1)^{R}$ in the definition of the twisted index (3.3) since

$$
\exp \left(\oint_{S^{1}} A_{\mathrm{CFT}}^{\mathrm{Bolt}_{ \pm}}\right)=\exp \left(\int_{D_{2}} F_{\mathrm{CFT}}^{\mathrm{Bolt}_{ \pm}}\right)=\exp ( \pm i \pi)=-1 .
$$

In the Euclidean path integral of the twisted index, we impose anti-periodic boundary condition for fermionic fields, which is also compatible with the shrinking of the $S^{1}$-cycle in the bulk.

Using (3.26), we finally obtain

$$
\begin{aligned}
S_{\theta \text {-term }}\left[\operatorname{Bolt}_{ \pm}\right] & =-i \frac{N^{3}+\mathcal{O}(N)}{6} \times \frac{c s(M)}{2 \pi^{3} L^{2}} \times 2 \times\left(\int_{\Sigma_{g}} \frac{L}{2} \operatorname{vol}\left(\Sigma_{g}\right)\right) \times\left(\mp \frac{L}{2} \int_{D_{2}} \operatorname{vol}\left(D_{2}\right)\right) \\
& = \pm i \frac{N^{3}+\mathcal{O}(N)}{3 \pi}(1-g) \times c s(M)
\end{aligned}
$$

The above computation then holographically implies the proposal in (3.19).

\section{Acknowledgments}

We thank Nikolay Bobev, Anthony M. Charles, Kiril Hristov, Jinsung Park, Valentin Reys and especially Seok Kim for insightful comments and discussions. SC thanks APCTP for kind hospitality during his visiting, where part of this work was done. SC is partially supported by the National Research Foundation of Korea (NRF) Grant 2018R1A2B6004914 and by NRF-2017-Global Ph.D. Fellowship Program. The research of DG and NK is supported by the NRF Grant 2019R1A2C2004880. DG also acknowledges support by the appointment to the JRG program at the APCTP through the Science and Technology Promotion Fund and Lottery Fund of the Korean Government, as well as support by the Korean Local Governments, Gyeongsangbuk-do Province, and Pohang City.

\section{A Global structure of Bolt solutions}

For the computation of the topological $\theta$-term in (3.23), we consider a non-supersymmetric deformation of the Bolt \pm solutions with $p=0$, where the topological structure becomes more manifest. The deformed Euclidean background for $g>1$ is given as follows [22, 52]

$$
\begin{aligned}
d s^{2} / L^{2} & =\lambda(\rho)(d \tau-2 s \cosh \theta d \phi)^{2}+\frac{d \rho^{2}}{\lambda(\rho)}+\left(\rho^{2}-s^{2}\right) d s^{2}\left(\Sigma_{g}\right), \\
A_{\tau} & =\frac{L}{2 s} \frac{P\left(\rho^{2}+s^{2}\right)-2 s Q \rho}{\rho^{2}-s^{2}}, \quad A_{\phi}=-L \frac{P\left(\rho^{2}+s^{2}\right)-2 s Q \rho}{\rho^{2}-s^{2}} \cosh \theta .
\end{aligned}
$$

Here we define

$$
\lambda(\rho):=\frac{\left(\rho^{2}-s^{2}\right)^{2}+\left(-1-4 s^{2}\right)\left(\rho^{2}+s^{2}\right)-2 M \rho+P^{2}-Q^{2}}{\rho^{2}-s^{2}} .
$$


The supersymmetric Bolt \pm solutions with $p=0$ correspond to

$$
\begin{aligned}
P & =-2 s^{2}-\frac{1}{2}, \quad M=2 s Q_{ \pm}, \quad Q=Q_{ \pm}, \\
\text {with } Q_{ \pm} & =\mp \frac{16 s^{2} \sqrt{\left(16 s^{2}\right)^{2}+128 s^{2}}}{128 s^{2}} .
\end{aligned}
$$

We consider the deformed geometry with $s=\epsilon, M=0, P=-\frac{1}{2}$ with small $\epsilon>0$. When $\epsilon \rightarrow 0$ and $Q \rightarrow \mp 0$, the deformed geometries approach to the Bolt \pm geometries. In the limit, the deformed geometry simplified as

$$
\lambda(\rho)=\rho^{2}-1+\frac{\frac{1}{4}-Q^{2}}{\rho^{2}}+o\left(\epsilon^{2}\right), \quad F_{\rho \tau}=\frac{L Q}{\rho^{2}}+o(\epsilon), \quad F_{\Sigma_{g}}=\frac{L}{2} \operatorname{vol}\left(\Sigma_{g}\right)+o(\epsilon) .
$$

Note that $\rho \geq \rho_{0}:=\frac{\sqrt{1+2|Q|}}{\sqrt{2}}$. To avoid conical singularity at $\rho=\rho_{0}, \tau$ should be periodic with the following periodicity

$$
\Delta \tau=\frac{4 \pi}{\left|\lambda^{\prime}\left(\rho_{0}\right)\right|}=\frac{\pi \sqrt{|Q|+\frac{1}{2}}}{|Q|} .
$$

So the topology of the deformed geometry is $\mathcal{M}_{4}^{\text {Bolt }}{ }_{ \pm} \sim D_{2} \times \Sigma_{g}$, where $\rho$ parametrizes the radial direction of $D_{2}$. Along the $\Sigma_{g}$, magnetic flux $\operatorname{vol}\left(\Sigma_{g}\right)$ is turned on. To measure the flux along $D_{2}$, we compute

$$
\int_{D_{2}} F=\left(\int_{\rho_{0}}^{\infty} \frac{L Q}{\rho^{2}}\right) \times \Delta \tau=\frac{L Q}{\sqrt{|Q|+\frac{1}{2}}} \times \frac{\pi \sqrt{|Q|+\frac{1}{2}}}{|Q|}=\mp \pi L .
$$

Due to the topological property of the integral, the computation is exact in $\epsilon$. Thus, it implies that $F_{D_{2}} \sim \mp \frac{L}{2} \operatorname{vol}\left(D_{2}\right)$.

\section{B Numerical verification of the conjecture (3.21) with $M=\left(\boldsymbol{S}^{\mathbf{3}} \backslash \mathbf{4}_{\mathbf{1}}\right)_{P / Q}$}

Let $\left(S^{3} \backslash \mathbf{4}_{1}\right)_{P / Q}$ be a 3 -manifold obtained by a Dehn surgery along the figure-eight knot (denoted by $\mathbf{4}_{1}$ ) with a slope $P / Q \in \mathbb{Q}$. The manifold is hyperbolic except $P / Q \in$ $\{0, \pm 1, \pm 2, \pm 3, \pm 4\}$. Fundamental group of the manifold is given as follows

$$
\pi_{1} M=\left\langle a, b, \mathbf{m}, \mathbf{l}: a b^{-1} a^{-1} b a=b a b^{-1} a^{-1} b, \mathbf{m}=a, \mathbf{l}=a b^{-1} a b a^{-2} b a b^{-1} a^{-1}, \mathbf{m}^{P} \mathbf{l}^{Q}=1\right\rangle .
$$

For a given irreducible $\mathrm{SL}(2, \mathbb{C})$ flat connection $\rho \in \operatorname{Hom}\left(\pi_{1} M \rightarrow \mathrm{SL}(2, \mathbb{C})\right)$, its Ray-SingerReidemeister torsion $\mathbf{T o r}_{\tau_{2 n+1}}(\rho, \mathrm{SL}(2, \mathbb{C}))$ can be computed as follows [53]

$$
\begin{aligned}
& \operatorname{Tor}_{\tau_{2 n+1}}(\rho, \operatorname{SL}(2, \mathbb{C})) \\
& =\left(\frac{P\left(\ell-\frac{1}{\ell}\right) m^{4}}{\left(m^{4}-1\right)\left(4-2 m^{2}+4 m^{4}\right)}+Q\right) \frac{\lim _{t \rightarrow 1}(t-1)^{-1} \Delta\left(t ; \tau_{2 n+1}, \rho\right)}{\prod_{a=1}^{n}\left(1-m^{2 a R} \ell^{2 a}\right)\left(1-m^{-2 a R} \ell^{-2 a S}\right)}, \\
& \text { with } \Delta\left(t ; \tau_{2 n+1}, \rho\right):=\frac{\operatorname{det}\left(I_{2 n+1}-t^{-1} A_{n} B_{n}^{-1} A_{n}^{-1}+A_{n} B_{n}^{-1} A_{n}^{-1} B_{n}-t B_{n}+B_{n} A_{n} B_{n}^{-1} A_{n}^{-1}\right)}{\operatorname{det}\left(t I_{2 n+1}-B_{n}\right)} .
\end{aligned}
$$


Here $A_{n}:=\tau_{2 n+1}(\rho(a)), B_{n}:=\tau_{2 n+1}(\rho(b))$ and $I_{2 n+1}$ is the identity matrix of $(2 n+1) \cdot m$ and $\ell$ are defined in the following relation

$$
(\rho(\mathbf{m}), \rho(\mathbf{l})) \sim_{\text {conj }}\left(\left(\begin{array}{cc}
m & * \\
0 & m^{-1}
\end{array}\right),\left(\begin{array}{cc}
\ell & * \\
0 & \ell^{-1}
\end{array}\right)\right) .
$$

Here, two integers $R$ and $S$ are chosen such that $P S-Q R=1$.

$M=\left(S^{3} \backslash \mathbf{4}_{1}\right)_{P / Q=5}$ case $\quad$ The holonomy matrices for the flat connection $\rho_{N=2}^{\text {geom }}$ are $^{3}$

$$
\begin{aligned}
\rho_{N=2}^{\text {geom }}(a) & =\left(\begin{array}{cc}
0.58480+0.37948 i & 0 \\
-1 & 1.2033-0.7808 i
\end{array}\right) \\
\rho_{N=2}^{\text {geom }}(b) & =\left(\begin{array}{cc}
0.58480+0.37948 i & -0.99245+0.51312 i \\
0 & 1.2033-0.7808 i
\end{array}\right) .
\end{aligned}
$$

Then, its Ray-Singer-Reidemeister torsions are

$$
\begin{aligned}
&\left\{\log \mathbf{T o r}_{\tau_{2 n+1}}\left[\rho_{N=2}^{\text {geom }}\right]\right\}_{n=1}^{15} \quad(\bmod 2 \pi i) \\
&=\{1.66019-2.42623 i, 2.47618+1.40108 i, 4.36609-2.48725 i, 6.81514+1.25657 i, \\
&9.90447-0.16483 i, 13.75025-0.61606 i, 18.0928-0.1422 i, 23.0814+1.3424 i\} \\
& 28.7128-2.5184 i, 34.9561+0.8746 i, 41.8289-1.0394 i, 49.3278-1.9892 i, \\
&57.4486-1.9708 i, 66.1951-0.9834 i, 75.5668+0.9714 i\} .
\end{aligned}
$$

The above numerical values are compatible with the theorem in (3.14) and the conjecture in (3.21) since the following series well converges:

$$
\begin{aligned}
\left\{\log \operatorname{Tor}_{\tau_{2 n+1}}\left[\rho_{N=2}^{\text {geom }}\right]-\frac{\operatorname{vol}(M)}{\pi}\left(n^{2}+n+\frac{1}{6}\right)-i \frac{c s(M)}{\pi}\left(n^{2}+n\right)\right\}_{n=1}^{15}(\bmod 2 \pi i) \\
=\{0.983369+2.88886 i, 0.549836+4.77999 i, 0.56547+4.27058 i, 0.515488+4.14204 i, \\
\quad 0.481026+4.16337 i, 0.578252+4.18678 i, 0.547515+4.16725 i, 0.538042+4.19027 i\} \\
\quad 0.546595+4.18297 i, 0.542266+4.17826 i, 0.542725+4.18166 i, 0.544512+4.18118 i, \\
\quad 0.543498+4.18071 i, 0.543412+4.18129 i, 0.543708+4.18107 i\} .
\end{aligned}
$$

Here we used the fact that $\operatorname{vol}(M)=0.98137$ and $c s(M)=1.52067$ [54].

In the same way, we also numerically confirmed the conjecture for other examples of hyperbolic $M=\left(S^{3} \backslash \mathbf{4}_{1}\right)_{P / Q}$.

Open Access. This article is distributed under the terms of the Creative Commons Attribution License (CC-BY 4.0), which permits any use, distribution and reproduction in any medium, provided the original author(s) and source are credited.

\footnotetext{
${ }^{3}$ There are only 4 irreducible $\mathrm{SL}(2, \mathbb{C})$ flat connections on the 3-manifold. Two of them are real, i.e. trace of all holonomy matrices are real, and the other twos correspond to $\mathcal{A}_{N=2}^{\text {geom }}$ and $\mathcal{A}_{N=2}^{\overline{\text { geom }}}$. The notion of being $\mathcal{A}_{N=2}^{\text {geom }}$ or $\mathcal{A}_{N=2}^{\overline{\text { geom }}}$ depends on the orientation choice of $M$ and we in particular choose one choice.
} 


\section{References}

[1] J.M. Maldacena, The large $N$ limit of superconformal field theories and supergravity, Int. J. Theor. Phys. 38 (1999) 1113 [InSPIRE].

[2] J. Kinney, J.M. Maldacena, S. Minwalla and S. Raju, An index for 4 dimensional super conformal theories, Commun. Math. Phys. 275 (2007) 209 [hep-th/0510251] [INSPIRE].

[3] F. Benini, K. Hristov and A. Zaffaroni, Black hole microstates in AdS $S_{4}$ from supersymmetric localization, JHEP 05 (2016) 054 [arXiv:1511.04085] [INSPIRE].

[4] F. Benini and A. Zaffaroni, Supersymmetric partition functions on Riemann surfaces, Proc. Symp. Pure Math. 96 (2017) 13 [arXiv:1605.06120] [INSPIRE].

[5] F. Benini and A. Zaffaroni, A topologically twisted index for three-dimensional supersymmetric theories, JHEP 07 (2015) 127 [arXiv: 1504.03698] [INSPIRE].

[6] C. Closset and H. Kim, Comments on twisted indices in $3 d$ supersymmetric gauge theories, JHEP 08 (2016) 059 [arXiv: 1605.06531] [INSPIRE].

[7] S. Choi, J. Kim, S. Kim and J. Nahmgoong, Large AdS black holes from QFT, arXiv:1810.12067 [INSPIRE].

[8] F. Benini and P. Milan, Black holes in $4 D N=4$ super-Yang-Mills field theory, Phys. Rev. X 10 (2020) 021037 [arXiv: 1812.09613] [INSPIRE].

[9] S. Choi, C. Hwang and S. Kim, Quantum vortices, M2-branes and black holes, arXiv: 1908.02470 [INSPIRE].

[10] S. Choi and S. Kim, Large $A d S_{6}$ black holes from $C F T_{5}$, arXiv:1904.01164 [INSPIRE].

[11] P. Agarwal, S. Choi, J. Kim, S. Kim and J. Nahmgoong, AdS black holes and finite N indices, Phys. Rev. D 103 (2021) 126006 [arXiv:2005.11240] [INSPIRE].

[12] A. Cabo-Bizet, D. Cassani, D. Martelli and S. Murthy, Microscopic origin of the Bekenstein-Hawking entropy of supersymmetric AdS $S_{5}$ black holes, JHEP 10 (2019) 062 [arXiv: 1810.11442] [INSPIRE].

[13] D. Cassani and L. Papini, The BPS limit of rotating AdS black hole thermodynamics, JHEP 09 (2019) 079 [arXiv: 1906.10148] [INSPIRE].

[14] T. Dimofte, S. Gukov and L. Hollands, Vortex counting and Lagrangian 3-manifolds, Lett. Math. Phys. 98 (2011) 225 [arXiv:1006.0977] [InSPIRE].

[15] Y. Terashima and M. Yamazaki, SL $(2, R)$ Chern-Simons, Liouville, and gauge theory on duality walls, JHEP 08 (2011) 135 [arXiv:1103.5748] [INSPIRE].

[16] T. Dimofte, D. Gaiotto and S. Gukov, Gauge theories labelled by three-manifolds, Commun. Math. Phys. 325 (2014) 367 [arXiv:1108.4389] [INSPIRE].

[17] D. Gang, N. Kim and L.A. Pando Zayas, Precision microstate counting for the entropy of wrapped M5-branes, JHEP 03 (2020) 164 [arXiv: 1905.01559] [INSPIRE].

[18] L.J. Romans, Supersymmetric, cold and lukewarm black holes in cosmological Einstein-Maxwell theory, Nucl. Phys. B 383 (1992) 395 [hep-th/9203018] [INSPIRE].

[19] M.M. Caldarelli and D. Klemm, Supersymmetry of anti-de Sitter black holes, Nucl. Phys. B 545 (1999) 434 [hep-th/9808097] [INSPIRE].

[20] F. Azzurli, N. Bobev, P.M. Crichigno, V.S. Min and A. Zaffaroni, A universal counting of black hole microstates in $A d S_{4}$, JHEP 02 (2018) 054 [arXiv: 1707.04257] [INSPIRE]. 
[21] D. Gang and N. Kim, Large $N$ twisted partition functions in 3d-3d correspondence and holography, Phys. Rev. D 99 (2019) 021901 [arXiv:1808.02797] [InSPIRE].

[22] C. Toldo and B. Willett, Partition functions on $3 d$ circle bundles and their gravity duals, JHEP 05 (2018) 116 [arXiv:1712.08861] [INSPIRE].

[23] M. Pernici, K. Pilch and P. van Nieuwenhuizen, Gauged maximally extended supergravity in seven-dimensions, Phys. Lett. B 143 (1984) 103 [INSPIRE].

[24] J.P. Gauntlett, N. Kim and D. Waldram, M-fivebranes wrapped on supersymmetric cycles, Phys. Rev. D 63 (2001) 126001 [hep-th/0012195] [inSPIRE].

[25] A. Donos, J.P. Gauntlett, N. Kim and O. Varela, Wrapped M5-branes, consistent truncations and AdS/CMT, JHEP 12 (2010) 003 [arXiv: 1009.3805] [INSPIRE].

[26] H. Nastase, D. Vaman and P. van Nieuwenhuizen, Consistent nonlinear KK reduction of $11 D$ supergravity on $A d S_{7} \times S^{4}$ and selfduality in odd dimensions, Phys. Lett. B 469 (1999) 96 [hep-th/9905075] [INSPIRE].

[27] H. Nastase, D. Vaman and P. van Nieuwenhuizen, Consistency of the $A d S_{7} \times S^{4}$ reduction and the origin of selfduality in odd dimensions, Nucl. Phys. B 581 (2000) 179 [hep-th/9911238] [INSPIRE].

[28] M. Cvetič, H. Lü, C.N. Pope, A. Sadrzadeh and T.A. Tran, $S^{3}$ and $S^{4}$ reductions of type IIA supergravity, Nucl. Phys. B 590 (2000) 233 [hep-th/0005137] [INSPIRE].

[29] W.D. Neumann and J. Yang, Bloch invariants of hyperbolic 3-manifolds, Duke Math. J. 96 (1999) 29.

[30] S. Choi, J. Kim, S. Kim and J. Nahmgoong, Comments on deconfinement in AdS/CFT, arXiv: 1811.08646 [INSPIRE].

[31] C. Romelsberger, Counting chiral primaries in $N=1, d=4$ superconformal field theories, Nucl. Phys. B $\mathbf{7 4 7}$ (2006) 329 [hep-th/0510060] [INSPIRE].

[32] S. Murthy, The growth of the $\frac{1}{16}-B P S$ index in $4 d N=4 S Y M$, arXiv:2005.10843 [INSPIRE].

[33] J.B. Gutowski and H.S. Reall, Supersymmetric AdS 5 black holes, JHEP 02 (2004) 006 [hep-th/0401042] [INSPIRE].

[34] J.B. Gutowski and H.S. Reall, General supersymmetric AdS $S_{5}$ black holes, JHEP 04 (2004) 048 [hep-th/0401129] [INSPIRE].

[35] Z.W. Chong, M. Cvetič, H. Lü and C.N. Pope, Five-dimensional gauged supergravity black holes with independent rotation parameters, Phys. Rev. D 72 (2005) 041901 [hep-th/0505112] [INSPIRE].

[36] H.K. Kunduri, J. Lucietti and H.S. Reall, Supersymmetric multi-charge AdS $S_{5}$ black holes, JHEP 04 (2006) 036 [hep-th/0601156] [INSPIRE].

[37] S. Choi and C. Hwang, Universal 3d Cardy block and black hole entropy, JHEP 03 (2020) 068 [arXiv: 1911.01448] [INSPIRE].

[38] N. Bobev and P.M. Crichigno, Universal spinning black holes and theories of class $\mathcal{R}$, JHEP 12 (2019) 054 [arXiv: 1909.05873] [INSPIRE].

[39] F. Benini, D. Gang and L.A. Pando Zayas, Rotating black hole entropy from M5 branes, JHEP 03 (2020) 057 [arXiv:1909.11612] [INSPIRE]. 
[40] J. Kim, S. Kim and J. Song, A 4d N=1 Cardy formula, JHEP 01 (2021) 025 [arXiv: 1904.03455] [INSPIRE].

[41] A. González Lezcano and L.A. Pando Zayas, Microstate counting via Bethe Ansätze in the 4d $N=1$ superconformal index, JHEP 03 (2020) 088 [arXiv:1907.12841] [INSPIRE].

[42] P.M. Crichigno and D. Jain, The 5d superconformal index at large $N$ and black holes, JHEP 09 (2020) 124 [arXiv:2005.00550] [INSPIRE].

[43] J. Nahmgoong, 6d superconformal Cardy formulas, JHEP 02 (2021) 092 [arXiv: 1907.12582] [INSPIRE].

[44] T. Dimofte, M. Gabella and A.B. Goncharov, $K$-decompositions and $3 d$ gauge theories, JHEP 11 (2016) 151 [arXiv: 1301.0192] [INSPIRE].

[45] D. Gang and K. Yonekura, Symmetry enhancement and closing of knots in $3 d / 3 d$ correspondence, JHEP 07 (2018) 145 [arXiv: 1803.04009] [INSPIRE].

[46] G.Y. Cho, D. Gang and H.-C. Kim, M-theoretic genesis of topological phases, JHEP 11 (2020) 115 [arXiv : 2007.01532] [InSPIRE].

[47] J. Cheeger, Analytic torsion and Reidemeister torsion, Proc. Nat. Acad. Sci. 74 (1977) 2651.

[48] K. Reidemeister, Homotopieringe und Linsenräume (in German), Abh. Math. Semin. Univ. Hambg. 11 (1935) 102.

[49] $R$ torsion and the Laplacian on Riemannian manifolds, Adv. Math. 7 (1971) 145 [INSPIRE].

[50] J. Park, Reidemeister torsion, complex volume, and Zograf infinite product for hyperbolic 3-manifolds with cusps, arXiv:1712.08270.

[51] N. Bobev, A.M. Charles, K. Hristov and V. Reys, The unreasonable effectiveness of higher-derivative supergravity in AdS $S_{4}$ holography, Phys. Rev. Lett. 125 (2020) 131601 [arXiv: 2006.09390] [INSPIRE].

[52] N. Bobev, A.M. Charles, D. Gang, K. Hristov and V. Reys, Higher-derivative supergravity, wrapped M5-branes, and theories of class $\mathcal{R}$, JHEP 04 (2021) 058 [arXiv:2011.05971] [INSPIRE].

[53] H. Goda, Twisted Alexander invariants and hyperbolic volume, Proc. Jpn. Acad. A 93 (2017) 61 [arXiv: 1604.07490].

[54] M. Culler, N.M. Dunfield, M. Goerner and J.R. Weeks, SnapPy, a computer program for studying the geometry and topology of 3-manifolds, http://snappy.computop.org. 